\title{
optica
}

\section{Enhancement of optical forces using slow light in a photonic crystal waveguide: supplementary material}

\author{
Mark G. Scullion ${ }^{1, *}$, Yoshihiko Arita ${ }^{2}$, Thomas F. Krauss ${ }^{1}$, and Kishan \\ DHOLAKIA 2,* \\ ${ }^{1}$ Department of Physics, University of York, York, YO10 5DD, United Kingdom \\ ${ }^{2}$ SUPA, School of Physics \& Astronomy, University of St Andrews, North Haugh, St Andrews, KY16 9SS, United Kingdom \\ *Corresponding author: mark.scullion@york.ac.uk orkd1@st-andrews.ac.uk
}

Published 17 September 2015

\begin{abstract}
This document provides supplementary information to "Enhancement of optical forces using slow light in a photonic crystal waveguide," http://dx.doi.org/10.1364/optica.2.000816. (C) 2015

Optical Society of America

http://dx.doi.org/10.1364/optica.2.000816.s001
\end{abstract}

\section{SUPPLEMENTARY NOTE 1}

We used the Finite Difference Time Domain (FDTD) methods (MEEP [1]) to simulate the forces acting on a $490 \mathrm{~nm}$ particle in 3D. For the photonic crystal we used a period of $a=420 \mathrm{~nm}$, radius $r=0.316 a$, slab thickness $220 \mathrm{~nm}$ and a grid size of $\lambda / 90$. The polystyrene particle was assumed to have a refractive index of 1.57. The simulated force enhancement (shown in Fig. 5 of the main text) was computed using force regions surrounding the particle, and was normalized to the same bead on top of a waveguide of width $\sqrt{3} a$ (i.e. the same width as a W1 waveguide, but without the surrounding mirror holes). The corresponding transmission spectrum, also simulated in MEEP with the same parameters and normalized to a waveguide, is shown in Fig. S1a.

In addition to the MEEP simulations, the simulated group index was computed using MPB [2] in 3D using the same parameters as the MEEP simulations and is shown in Fig. S1b.

A simulation of a $200 \mathrm{~nm}$ particle was also performed. The results are shown in Fig. S2. Fig. S2a shows the force normalized to a $200 \mathrm{~nm}$ bead on top of a waveguide, whilst Fig. S2b shows the force relative to a $490 \mathrm{~nm}$ bead on top of the same photonic crystal.

\section{SUPPLEMENTARY NOTE 2}

The force enhancement in an air filled photonic crystal was also modeled relative to a waveguide. In this case the hole radius was reduced to $r=0.295 a$ to give operation around $1550 \mathrm{~nm}$. The results are shown in Fig. S3.

\section{SUPPLEMENTARY NOTE 3}

We investigated the particle dynamics with the particles of two different sizes ( $400 \mathrm{~nm}$ and $600 \mathrm{~nm}$ in diameter) and with
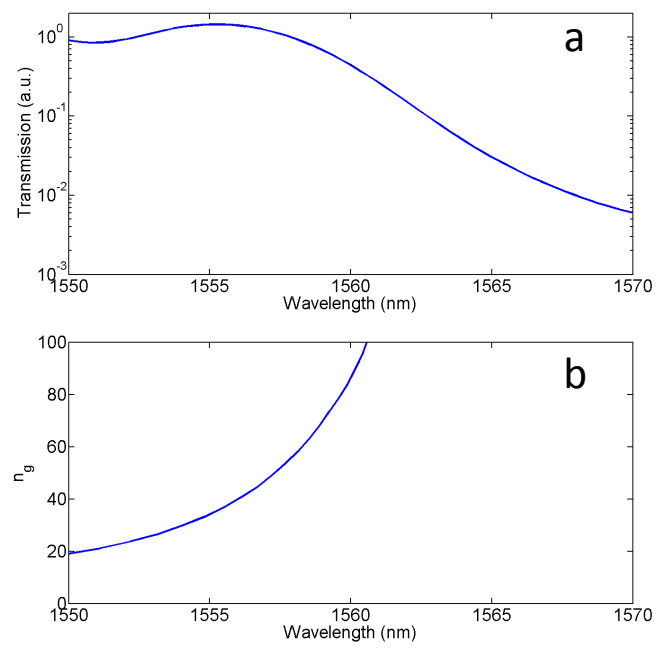

Fig. S1. Simulated slow light profile for a photonic crystal waveguide. a) Simulated transmission spectrum (MEEP). b) Simulated group index (MPB).

two guided optical powers of $2.5 \mathrm{~mW}$ and $4 \mathrm{~mW}$, respectively (Fig. S4). As the scattering force scales with $\sim r^{6}$ while the Stokes drag force scales linearly with $r$, where $r$ is the radius of the particle, the terminal velocity will increase with the particle size [3].

First we observe that the particle velocity scales with the guided optical power. The maximum velocities were measured at around $1560 \mathrm{~nm}$ for all cases. The larger particles of $600 \mathrm{~nm}$ gained more velocity for every wavelength probed due to the higher scattering forces relative to the Stokes drag force. 

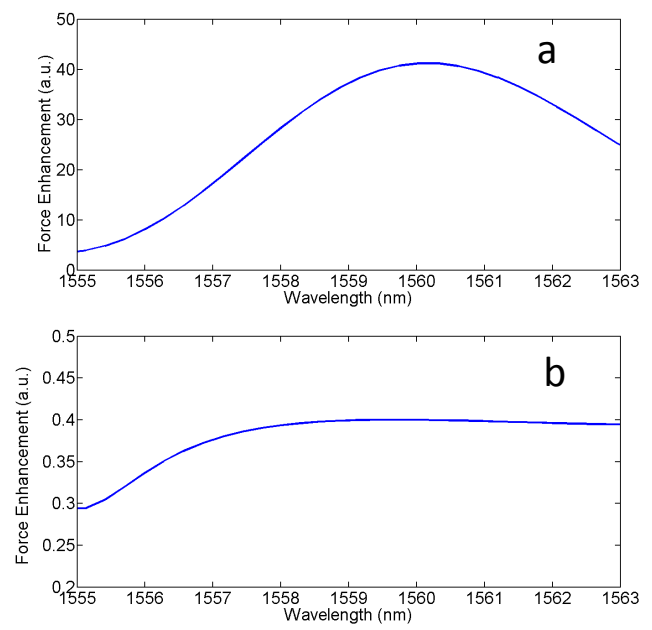

Fig. S2. Force enhancement simulation for a $200 \mathrm{~nm}$ bead. a) Normalized to a $200 \mathrm{~nm}$ bead on top of a waveguide. b) Normalized to a $490 \mathrm{~nm}$ bead on top of a photonic crystal.
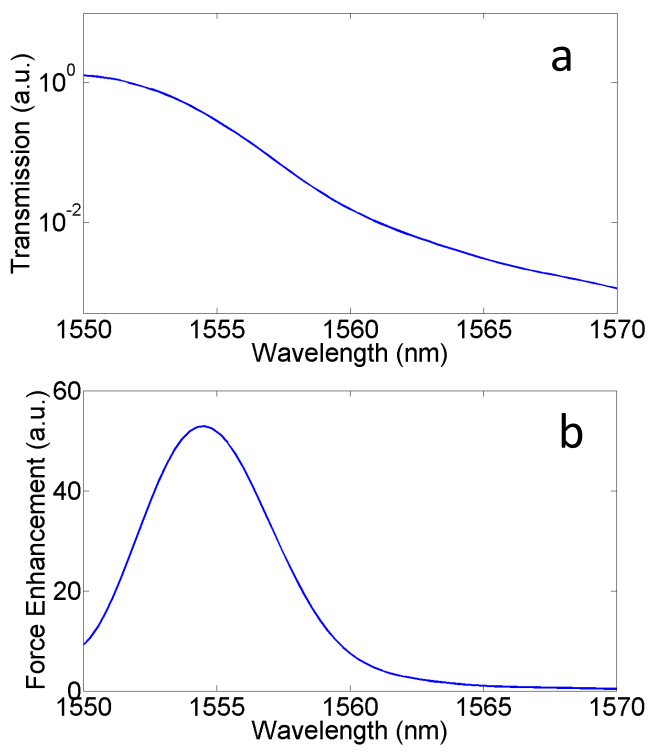

Fig. S3. Modelling of particle on top of an air filled photonic crystal waveguide. a) Simulated transmission spectrum (MEEP) in air filled photonic crystal. b) Corresponding simulated force enhancement relative to a waveguide.

Note that in Fig. S4 the guiding velocity appears to rise again after $1561 \mathrm{~nm}$. This rise is also mirrored in the corresponding trap stiffness measurements shown in Fig 4 within the main text. This rise may be due to variations in the waveguide transmission relative to the simulations as a result of fabrication errors and stuck particles from the earlier experiments.

\section{REFERENCES}

1. A. F. Oskooi, D. Roundy, M. Ibanescu, P. Bermel, J. D. Joannopoulos, and S. G. Johnson, "Meep: A flexible freesoftware package for electromagnetic simulations by the fdtd method," Comput. Phys. Commun. 181, 687-702 (2010).

2. S. G. Johnson and J. D. Joannopoulos, "Block-iterative frequency-domain methods for maxwell's equations in a planewave basis," Opt. Express 8, 173-190 (2001).

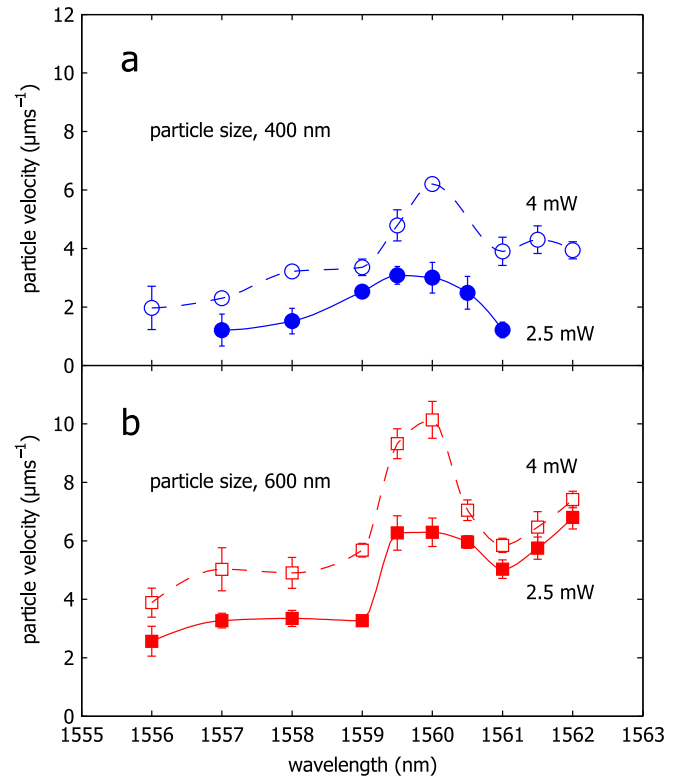

Fig. S4. Particle velocities for different particle sizes and optical powers. a) $400 \mathrm{~nm}$ size particles b) $600 \mathrm{~nm}$ size particles.

3. K. C. Neuman and S. M. Block, "Optical trapping," Rev. Sci. Instrum. 75, 2787-2809 (2004). 